OPEN ACCESS

Edited by:

Rakesh Sharma,

Institute of Genomics and Integrative

Biology (CSIR), India

Reviewed by:

Alan Leonard,

Florida Institute of Technology, USA

Baojun Wu,

Wayne State University, USA

*Correspondence:

Kok-Gan Chan

kokgan@um.edu.my

Specialty section: This article was submitted to

Evolutionary and Genomic

Microbiology,

a section of the journal

Frontiers in Microbiology

Received: 30 June 2016 Accepted: 26 September 2016

Published: 14 October 2016

Citation:

Yong $D$, Tee $K K$, Yin $W-F$ and

Chan K-G (2016) Characterization and Comparative Overview of Complete Sequences of the First Plasmids of

Pandoraea across Clinical and Non-clinical Strains

Front. Microbiol. 7:1606.

doi: 10.3389/fmicb.2016.01606

\section{Characterization and Comparative Overview of Complete Sequences of the First Plasmids of Pandoraea across Clinical and Non-clinical Strains}

\author{
Delicia Yong ${ }^{1}$, Kok Keng Tee ${ }^{1}$, Wai-Fong Yin ${ }^{2}$ and Kok-Gan Chan ${ }^{2 *}$ \\ ${ }^{1}$ Department of Medical Microbiology, Faculty of Medicine, University of Malaya, Kuala Lumpur, Malaysia, ${ }^{2}$ Division of \\ Genetics and Molecular Biology, Faculty of Science, Institute of Biological Sciences, University of Malaya, Kuala Lumpur, \\ Malaysia
}

To date, information on plasmid analysis in Pandoraea spp. is scarce. To address the gap of knowledge on this, the complete sequences of eight plasmids from Pandoraea spp. namely Pandoraea faecigallinarum DSM 23572 ${ }^{\top}$ (pPF72-1, pPF72-2), Pandoraea oxalativorans DSM 23570 (pPO70-1, pPO70-2, pPO70-3, pPO70-4), Pandoraea vervacti NS15 (pPV15) and Pandoraea apista DSM 16535 (pPA35) were studied for the first time in this study. The information on plasmid sequences in Pandoraea spp. is useful as the sequences did not match any known plasmid sequence deposited in public databases. Replication genes were not identified in some plasmids, a situation that has led to the possibility of host interaction involvement. Some plasmids were also void of par genes and intriguingly, repA gene was also not discovered in these plasmids. This further leads to the hypothesis of host-plasmid interaction. Plasmid stabilization/stability protein-encoding genes were observed in some plasmids but were not established for participating in plasmid segregation. Toxin-antitoxin systems MazEF, VapBC, RelBE, YgiT-MqsR, HigBA, and ParDE were identified across the plasmids and their presence would improve plasmid maintenance. Conjugation genes were identified portraying the conjugation ability amongst Pandoraea plasmids. Additionally, we found a shared region amongst some of the plasmids that consists of conjugation genes. The identification of genes involved in replication, segregation, toxin-antitoxin systems and conjugation, would aid the design of drugs to prevent the survival or transmission of plasmids carrying pathogenic properties. Additionally, genes conferring virulence and antibiotic resistance were identified amongst the plasmids. The observed features in the plasmids shed light on the Pandoraea spp. as opportunistic pathogens.

Keywords: conjugation, toxin-antitoxin systems, virulence genes, antibiotic resistance, plasmid 


\section{INTRODUCTION}

Extrachromosomal DNA provides great impact in the evolution of bacteria in adapting to their surroundings. Depending on the genes it carries, it could act as a fitness factor to the host, or serve as a virulence factor by transforming the host into a pathogen. The impact is enhanced if coupled with the presence of functional genes for antibiotic resistance. Virulence factors enable bacteria to defend themselves against host defense systems whereas antibiotic resistance allows them to combat against antimicrobial treatment (Beceiro et al., 2013). Plasmids are one of the primary sources for extrachromosomal DNA in bacteria and are capable of self-transmission. Most importantly, plasmids were identified as leading factors to outbreaks such as the case for plasmid p1658/97 that contributed to $\beta$-lactam antibiotics resistance in Escherichia coli isolates during the clonal outbreak in a hospital in Warsaw, Poland (Zienkiewicz et al., 2007). In another situation, the outbreak of NDM-1-producing Klebsiella pneumoniae at the Kunning City Maternal and Child health hospital in China, may have been caused by the bla $a_{\mathrm{NDM}-1^{-}}$ harboring plasmid that was present in all the clinical isolates (Zheng et al., 2016). Hence, the spread of virulence factors and antibiotic resistance properties caused by plasmids is possible, leading to a threat in public health.

Pandoraea (P.) genus consists of nine species namely P. apista, P. pnomenusa, P. pulmonicola, P. sputorum, $P$. vervacti, $P$. oxalativorans, $P$. thiooxydans, $P$. norimbergensis, and P. faecigallinarum (Coenye et al., 2000; Anandham et al., 2010; Sahin et al., 2011) with rod-shape, aerobic and gramnegative properties. Non-clinical strains have been isolated from soil, chicken dung and oxic water layer (Coenye et al., 2000; Anandham et al., 2010; Sahin et al., 2011). Little is known about the pathogenic potential of these strains. Clinical strains have been isolated from cystic fibrosis (CF) patients (Coenye et al., 2000) as well as non-CF patients (Stryjewski et al., 2003; Pimentel and MacLeod, 2008). There is very limited information on the factors contributing to the pathogenicity potential in Pandoraea. It is also not known if the virulence of Pandoraea is linked to the existence of plasmids. Previous studies have reported on the contribution of plasmids to the virulence of members of Burkholderia cepacia complex (BCC) (Agnoli et al., 2014) and Mycobacterium avium-intracellulare (MAI) complex (Hellyer et al., 1991). Similar to Pandoraea, both these bacterial groups are opportunistic pathogens (Mahenthiralingam et al., 2008; Whiley et al., 2015). This leads to a speculation that the pathogenicity of Pandoraea could be contributed by the presence of plasmids.

Four completely sequenced Pandoraea strains harbored eight plasmids in total. Along with five previously reported plasmids from P. oxalativorans DSM 23570 ${ }^{\mathrm{T}}$ (pPO70-1, pPO70-2, pPO703, pPO70-4) (Chan et al., 2016) and P. vervacti NS15 (pPV15) (Ee et al., 2015), three novel plasmids were sequenced for comparison in this study. Here, we present the first comparative study of plasmids within the Pandoraea genus. Our aims are to annotate and study their properties for replication, segregation, conjugation and presence of toxin-antitoxin (TA) systems. These systems have been reported responsible in maintaining the survival and replication of plasmids (Thomas, 2000; Kroll et al.,
2010; Baxter and Funnell, 2014). Knowledge on these systems is vital in disease treatment in which drug design can be employed (Sengupta and Austin, 2011). Additionally, we also narrowed our search for virulence and antibiotic resistance characteristics within the plasmids. The identification and study of plasmids are important to discover the features of novel plasmids, especially in opportunistic pathogens such as Pandoraea. These sequence data analyses will hopefully serve as a basis for future research.

\section{MATERIALS AND METHODS}

\section{Bacterial Strains, Plasmids, Culture Conditions, and Sequencing}

The Pandoraea species isolates $P$. faecigallinarum DSM $23572^{\mathrm{T}}$ and $P$. apista DSM $16535^{\mathrm{T}}$ were obtained from the Leibniz Institute DSMZ-German Collection of Microorganisms and Cell Cultures $\mathrm{GmbH}$. The isolates were cultured on Luria-Bertani medium for $24 \mathrm{~h}$ at $28^{\circ} \mathrm{C}$ with shaking at $220 \mathrm{rpm}$ in reference to a previous protocol (Lim et al., 2014). Genomic DNA was isolated using an Epicentre Masterpure DNA extraction kit (Epicentre, Inc., Madison, WI, USA) according to the manufacturer's instructions. To obtain complete genome sequences for a better genomic overview, Pacific Biosciences (PacBio) RS II Single Molecule Real Time (SMRT) sequencing method (Pacific Biosciences, Menlo Park, CA) was employed. Genomic DNA was processed into SMRTbell ${ }^{\mathrm{TM}}$ library using the SMRTbell template preparation kit 1.0 according to the "Procedure and checklist-20 kb template preparation using BluePippin sizeselection system" protocol as reported by Lim et al. (2016). Sequencing of the SMRTbell ${ }^{\mathrm{TM}}$ libraries were conducted using P6 DNA polymerase (C4 chemistry) which is PacBio's latest DNA sequencing chemistry that improves the average read length (http://investor.pacificbiosciences.com/releasedetail.cfm? releaseid=876252). Quality filtering and de novo assembly of the sequenced reads were performed using the Hierarchical Genome Assembly Process (HGAP) version 2 of PacBio (DevNet; Pacific Biosciences).

Based on the PacBio Circularizing and Trimming wiki at (https://github.com/PacificBiosciences/Bioinformatics-Training/ wiki/Circularizing-and-trimming), circularity of all contigs generated from the assembly was determined based on reads mapping across the beginning and end of the contigs. To confirm this, Gepard (Krumsiek et al., 2007) was used to generate dot plots for each contig to detect the presence of self-similar region at the $5^{\prime}$ and $3^{\prime}$ contig ends. The contigs were then subjected to PacBio Quiver algorithm for polishing prior to further analysis. Chromosomal contigs were determined based on the similarity of the contig sizes to the expected chromosomal size of Pandoraea and were further confirmed by using BLASTN. Subsequently, all extra-chromosomal contigs were aligned against the chromosomal contigs and were assessed using Contiguity (Sullivan et al., 2015). Only contigs which demonstrated low or no similarity to their respective chromosomal contigs were determined as plasmid contigs.

As a result, three plasmid contigs were observed. Non-clinical isolate $P$. faecigallinarum DSM $23572^{\mathrm{T}}$ harbors two plasmids 
(pPF72-1, pPF72-2) whereas clinical isolate P. apista DSM $16535^{\mathrm{T}}$ carries a single plasmid (pPA35). Complete sequences of plasmids from non-clinical strains $P$. oxalativorans DSM $23570^{\mathrm{T}}$ (pPO70-1, pPO70-2, pPO70-3, pPO70-4) (Chan et al., 2016) and P. vervacti NS15 (pPV15) (Ee et al., 2015) were obtained from NCBI.

\section{Bioinformatics Analysis}

Prokaryotic Dynamic Programming Genefinding Algorithm (Prodigal) Version 2.6.2 (Hyatt et al., 2010) was used in the prediction of open reading frames. The predicted sequences were then functionally annotated by performing homology search against the NCBI nr database, InterProScan (Quevillon et al., 2005) and the Conserved Domain Database (CDD) (MarchlerBauer et al., 2015). Identification of key genes conferring biological advantages were performed based on Virulence Factors Database (VFDB) (Chen et al., 2005), The Comprehensive Antibiotic Resistance Database (CARD) (McArthur et al., 2013) and TAfinder (http://202.120.12.133/TAfinder/index.php).

\section{Nucleotide Sequence Accession Numbers}

The accession numbers of the complete sequences of the three plasmids sequenced in this study and of the five plasmids from NCBI can be found in GenBank with the accession numbers CP011808 (pPF72-1), CP011809 (pPF72-2), CP013482 (pPA35), СР011518 (pPO70-1), CP011519 (pPO70-2), СР011520 (pPO70-3), CP011521 (pPO70-4), and CP010898 (pPV15).

\section{RESULTS AND DISCUSSION}

\section{General Features of Plasmids}

All eight plasmids range in sizes from 46,278 to 640,227 $\mathrm{bp}$, organized in a single contig with overall $\mathrm{G}+\mathrm{C}$ content in the range of $57.81-63.8 \%$. The number of open reading frames in the range of $65-626$ was predicted across the plasmids in which 62-591 are protein-encoding genes (Supplementary Figures S1-S4 and Supplementary Table S1). The features of each plasmid are shown in Table 1. The putative origin of vegetative replication (oriV) and terminus (terV) for each plasmid were identified based on cumulative GCskew analysis using GenSkew (http://genskew.csb.univie.ac.at/) (Supplementary Figures S5-S8). The predicted oriV and terV are marked by the minimum and maximum values of the cumulative GC-skew, respectively, which corresponds to the nucleotide positions (Grigoriev, 1998). Overall, Pandoraea plasmid sequences yielded low similarity against plasmid sequences from the RefSeq and EMBL nucleotide sequence database with sequence completeness of $<12 \%$. In addition, no homologous gene organization was observed. Hence, no comparison between regions of the Pandoraea plasmids and other known plasmids could be made. These findings indicate that the Pandoraea plasmid sequences were not reported before.

To determine the possible origins of genes within the Pandoraea plasmids, we performed a search against a nonplasmids database. The database was constructed based on bacterial genome sequences obtained from NCBI. We observed that majority of the genes across the plasmids matched to genomes of Burkholderia, Bordetella, Xanthomonas, Ralstonia, Pseudomonas, Alicycliphilus, Nitrosomonas and Cupriavidus with similarity and completeness scores well above $50 \%$.

\section{Origin of Vegetative Replication, Terminus, and Replication Genes}

Plasmid replication is vital in ensuring reproducibility and segregation of plasmids into daughter cells during host cell division. This is an essential aspect in the plasmid maintenance system and highlights the values of identifying the potential genes involved in replication machinery, especially in the unknown potential of plasmids in Pandoraea.

Putative replication initiator protein-encoding gene (repA) was identified in pPO70-4 and pPA35, indicating self-replication potential of these two plasmids. However, no repA was identified in the other plasmids. This has led us to speculate that a different replication mechanism exists in which a plasmid initiator protein is not employed, such as the case for plasmid ColE1 (del Solar et al., 1998) that reportedly uses DNA polymerase I for replication initiation.

Putative genes surrounding the putative predicted oriV and ter $V$ locations that could be involved in replication were identified. In pPA35, ori $V$ was identified at position 72,001 bp within an acyltransferase gene whereas ter $V$ was identified at position 31,001 bp within an intergenic region between conjugal transfer TraM containing domain protein and hypothetical protein-encoding genes. Conjugation tra and trb genes were found to flank either side of the terV. A similar observation was made in plasmid $\mathrm{pRF}$ in which its ter $V$ was flanked by conjugation genes (Gillespie et al., 2007). Other genes located downstream of the ter $V$ in pPA35 are partitioning genes (parA, parB) and repA. A single-strand DNA-binding (SSB) proteinencoding gene $(s s b)$ was found located next to repA and could be associated with DNA replication (Meyer and Laine, 1990).

The oriV at position 37,001 bp in pPO70-4 was found within an antitoxin gene. Located upstream are genes repA, ssb and a gene encoding for a partitioning protein which shares $96 \%$ identity with ParA of Xanthomonas. Another partitioning protein with a ParA domain was found downstream of the oriV and has $99 \%$ identity to the upstream partitioning protein-encoding gene. At position $25,001 \mathrm{bp}$, the ter $V$ is located within a type IV secretion system (T4SS) protein-encoding gene flanked on either side by conjugation genes. This is another feature similar to plasmid pRF (Gillespie et al., 2007).

As for pPF72-1, the oriV at position 250,000 bp is found within an intergenic region between parA and a hypothetical protein-encoding gene, a situation that matches the finding that parA is often located adjacent to oriV (Picardeau et al., 2000). The ter $V$ at position $332,001 \mathrm{bp}$ is located within a hypothetical protein-encoding gene. Plasmid pPF72-2 on the other hand, has its ori $V$ at position $124,001 \mathrm{bp}$ in an intergenic region between transposase and hypothetical protein-encoding genes whereas its ter $V$ at 30,001 bp is found within a hypothetical protein-encoding gene with $s s b$ located downstream.

Overall, only pPA35, pPF72-1, pPF72-2 and pPO70-4 showed the presence of genes that are possibly involved in their 
TABLE 1 | General plasmid features.

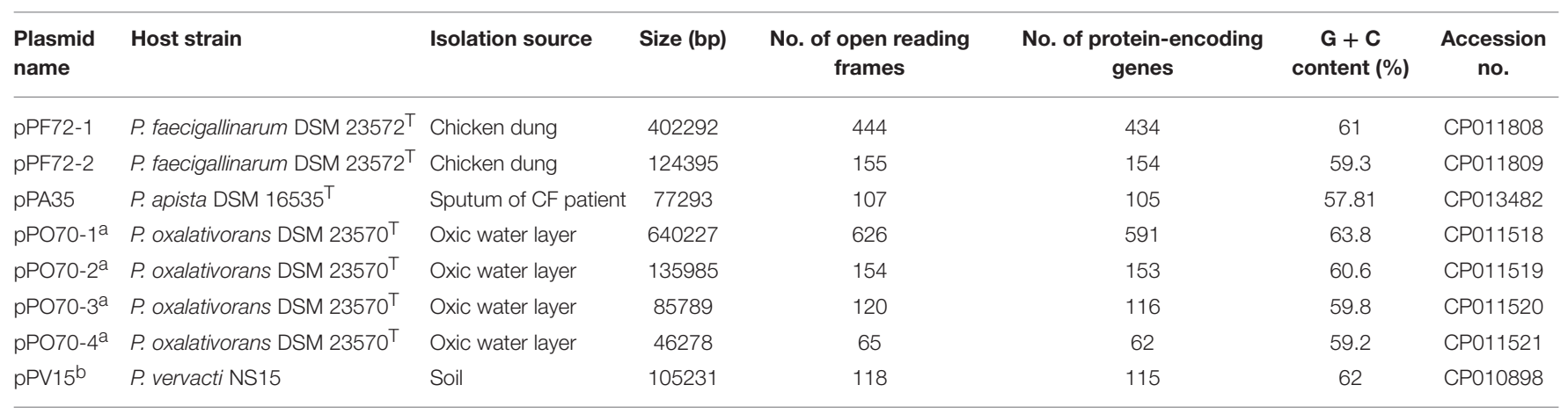

a From previously published data of P. oxalativoranss DSM 23570 ${ }^{T}$ genome (Chan et al., 2016).

${ }^{b}$ From previously published data of P. vervacti NS15 genome (Ee et al., 2015).

replication systems. Plasmids pPO70-1, pPO70-2, pPO70-3 and pPV15 showed no signs of replication genes located near the oriV and ter $V$ as well as in other parts of the plasmid. The observed characteristics raised the hypothesis of plasmid-host interaction for plasmid replication, as reported for plasmid Pps10 from Pseudomonas syringae (Fernández-Tresguerres et al., 1995). The absence of replication genes in the four plasmids motivates the understanding of the interaction between the plasmids and their respective host strains. The information can be potentially used to design the plasmids with a host-killing system, and enable them to be released into the environment as an effort to control possible infection by Pandoraea and other opportunistic pathogens.

\section{Partitioning Genes}

Partitioning genes function in the segregation of plasmids into daughter cells following plasmid replication. The segregation activity, that takes place as part of the second mechanism of plasmid maintenance, is also known as the partition system or par system. In the identification of putative partitioning genes, genes parA and parB of the type I par system (Gerdes et al., 2000) were observed in pPF72-1 and pPA35. These genes are trans-acting protein-encoding genes whereby parA encodes an ATPase that regulates the function of DNA binding protein-encoding parB (Bignell and Thomas, 2001). Besides the two aforementioned plasmids, pPO70-4 was found to contain two partitioning protein-encoding genes with a ParA domain identified in each gene.

No par genes were identified in pPF72-2, pPO70-1, pPO702, pPO70-3 and pPV15, indicating a possibly different mode of segregation besides the typical partition system. This situation is possible considering previous studies conducted on plasmids that describe the usage of other approaches for segregation other than the par system. One of these studies involves plasmid pSK1 from Staphylococcus aureus, in which an identified gene is involved in the increase of segregation stability while no ATPase activity was required to drive plasmid segregation (Simpson et al., 2003). In another study, plasmid R388 utilizes other genes instead of the par system for plasmid segregation (Guynet et al., 2011).

Interestingly, all the Pandoraea plasmids without par genes also did not contain repA. The consistent observations on this absence of replication and partitioning genes, strengthen the view on close interactions between the host strains and plasmids for the maintenance system. Plasmid stabilization/stability proteinencoding genes have been observed across pPF72-1, pPF72-2, pPO70-2, pPO70-3 and pPV15, possibly playing a part in plasmid segregation but this has not been confirmed.

\section{Toxin-Antitoxin Systems}

Toxin-antitoxin (TA) systems in plasmids form the third mechanism of plasmid maintenance. They have also been termed as post-segregational killing systems or plasmid addiction systems (Kroll et al., 2010). A single TA system consists of an antitoxin and a toxin protein. In the event that a daughter cell inherits a plasmid, the antitoxin protein acts to neutralize the action of the toxin, thereby preventing cell death (Sengupta and Austin, 2011). However, when plasmids are not distributed to daughter cells, the stable toxin protein causes cell death whereas the unstable antitoxin protein is degraded. In other words, TA systems prolong the presence of plasmids in the bacterial population by eliminating plasmid-free cells that have arisen due to unsuccessful plasmid replication and segregation. There are five classes of TA systems of which the class Type II is the best studied (Unterholzner et al., 2013). Hence, we have focused only on class Type II TA systems in our study.

The presence of putative genes known to behave as TA systems, have been identified across the Pandoraea plasmids except for pPV15. Supplementary Table S2 displays the list of TA systems found in each of the plasmids.

More than one TA system was observed in each of the plasmids except in pPO70-4. The observed presence of TA systems could be due to the nature of TA as a mobile element, and is subsequently integrated into the DNA of the plasmids. The presence of TA systems would enhance the post-segregational activity, and in turn ensure better plasmid maintenance. With the exception of pPO70-4, all plasmids in free-living strains have more TA systems as compared to pPA35, which originates from a host-associated strain. A study carried out by Pandey and Gerdes (2005) presented that free-living organisms possess multiple TA systems as compared to host-associated organisms that did not have any. This observation is similar to the Pandoraea plasmids from free-living strains, whereby the presence of TA 
systems proposes the need for survival in the constantly changing ecosystem. However, the identification of two TA systems in pPA35, suggests that the maintenance of this host-associated strain-harboring plasmid is still essential in adapting to an inconsistent environment.

A total of seven types of TA systems were observed across the Pandoraea plasmids namely MazEF, VapBC, RelBE, YgiTMqsR, HigBA and ParDE. The TA systems VapBC and RelBE of pPF72-1 and pPO70-3, respectively, were found within plasmid stabilization protein-encoding genes. It is proposed that these TA systems are helpful in preventing the loss of these elements in daughter cells. All plasmids except pPO70-2 and pPO70-4, have more than one copy of some of its TA systems. Such a situation will ensure a stable single-copy plasmid inheritance (Venkatesan et al., 2001). All TA systems identified in the Pandoraea plasmids were encoded near transposase or integrase encoding genes, indicating an ability to be mobilized by mobile genetic elements (Bustamante et al., 2014).

The orientation of type II TA systems is such that the antitoxin gene is situated upstream of the toxin gene (Van Melderen and Saavedra De Bast, 2009). However, we observed that some TA systems in the Pandoraea plasmids have an opposite orientation. This was observed amongst MazEF, VapBC, RelBE, HigBA and HipBA across pPF72-1, pPF72-2, pPO70-1 and pPO70-3, whereby RelBE appears to be the most frequent in number having an opposite orientation. Previous studies have also shown the same unusual occurrence in TA system orientation (Budde et al., 2007; Yamaguchi et al., 2009; Christensen-Dalsgaard et al., 2010).

Gene overlap in the TA systems has been observed across the Pandoraea plasmids. The occurrence of gene overlap in prokaryotes indicates translational coupling and this phenomenon is common in TA systems (Pandey and Gerdes, 2005). An overlap of 4 and 1 bp in TA systems were observed in pPF72-1, pPF72-2, pPO70-1, pPO70-2, pPO70-4 and pPA35. Generally, most TA systems have an overlap of $4 \mathrm{bp}$ or even $1 \mathrm{bp}$, in which the antitoxin stop codon overlaps the start codon of the toxin gene (Pandey and Gerdes, 2005). Other overlap sizes have also been observed amongst the Pandoraea plasmids. Overlaps of 7 and 5 bp were found within MazEF and RelBE, respectively, in pPF72-1. Plasmid pPF72-2 has 29, 11, and 20 bp overlaps in its RelBE whereas a 9 bp overlap is seen in HipBA in pPO70-1. Lastly, 13 and 29 bp overlaps were identified in RelBE in pPO703. Chan et al. (2012) have previously reported on other overlap sizes besides the usual 4 and 1 bp overlaps. Some TA systems in the Pandoraea plasmids have no gene overlap such as in pPF721, in which gaps of 118 bp (MazFike_domain, higA) and $47 \mathrm{bp}$ (RelBE) were observed whereas a gap of $170 \mathrm{bp}$ was identified within RelBE in pPO70-3.

\section{Conjugation}

Conjugation is one of the mechanisms of bacterial lateral gene transfer (Burrus and Waldor, 2004). Genes enabling conjugation are often located on plasmids and genetic transfer occurs when these conjugative plasmids are self-transmitted during bacterial cell to cell contact. The mode of conjugation is important as it enables plasmids to deliver features that can be beneficial to the host strains. An example has been reported by Rösch et al. (2014) where the presence of conjugative plasmid pLS20 conferred stress resistance to its host strain. The identification of the conjugation mechanism can also be helpful in preventing the transfer of pathogenic properties such as reported by Lujan et al. (2007), who described the disruption of relaxase to prevent antibiotic resistance transfer. The conjugative potential of Pandoraea plasmids has been predicted based on the presence of putative conjugation genes encoding T4SS components. Overall, these genes were predicted as tra, trb, trw and virB. These different gene designations exist overtime for different plasmids in particularly when they are categorized into different incompatibility groups (Stolz, 2014). Some of the examples of this categorization include tra, trb and trw of incompatibility groups IncF and IncN, IncP and IncW, respectively, as well as $\operatorname{vir} B$ of Ti plasmids (Christie, 1997). All these gene designations have been identified in the conjugation genes among the Pandoraea plasmids. No conjugation genes however, were identified in $\mathrm{pPO} 70-3$.

Conjugation genes in pPA35 and pPO70-4 were predicted as tra, trb and virB. In pPA35, two separate regions exist mainly coding for tra (tra region) and trb (trb region) genes, respectively. Similar regions were observed in plasmid pTiC58 of Agrobacterium tumefaciens (Alt-Mörbe et al., 1996). The tra region contains genes traC, traG, traJ and traL, in which all except traL are located on the lagging strand. Genes encoding a relaxase and peptidase S26, were also identified in this region in which the latter contains conjugal transfer peptidase TraF domain. An additional gene adjacent to the tra region was characterized with conjugal transfer protein TraM domain and is located on the leading strand. Genes in the $\operatorname{trb}$ region are $\operatorname{tr} b B, \operatorname{trb} C, \operatorname{tr} b D, \operatorname{tr} b G$, trbI, trbJ, trbL and traX. A single virB4 has also been identified in this region. Five additional genes were observed within and adjacent to the trb region with domains TrbA, TrbF, TrbH, TrbM and TrbN, respectively. For the trb region, the conjugation and additional genes were located on the leading strand except for the additional gene with conjugal transfer protein TrbA domain.

In contrast to the two conjugative regions in pPA35, pPO70-4 comprises a single conjugative region which contains genes $\operatorname{traC}$, trbL, traB, virB10 and virB3. Besides these, genes encoding pilus assembly protein $\mathrm{CpaF}$ and T4SS protein were also identified. Three additional genes were identified within and adjacent to the conjugative region of pPO70-4. These genes have domains TrbG, TrbF and T4SS lytic transglycosylase VirB1, respectively. Conjugation genes in pPO70-4 are all located on the lagging strand except for traC.

The best hit was identified for each additional gene via homology search with BLASTP against the $\mathrm{nr}$ database. Information on their domain and best hits are tabulated in Supplementary Table S3. They were found to carry conjugation domains and have close hits to conjugation genes suggesting that they are yet to be reported as conjugative-related genes.

In comparison to the conjugation genes in pPA35 and pPO70-4, the other Pandoraea plasmids contain differently annotated conjugation genes, except for a single Tra coupling protein-encoding gene $($ traD) found in pPO70-1, pPO70-2 and pPV15. These genes include $\operatorname{tr} w B$, virB11 and virB9. Relaxase, 
endonuclease and lytic transglycosylase and type VI secretion system (T6SS) protein-encoding genes were also identified.

Domain search revealed a T6SS lytic transglycosylase VirB1 domain within the lytic transglycosylase encoding genes, indicating that they could function in degrading the peptidoglycan wall in order to permit construction of structures such as the pilus during conjugation (Wallden et al., 2010). Furthermore, these genes have 59-65\% identity with T4SS protein VirB1 of Xanthomonas fuscans subsp. Aurantifolii str. ICPB 11122. According to Wang and Macrina (1995), the endonuclease, also known as the nickase, functions in carrying out a nick at the oriT during conjugation. It is suggestive that the endonuclease gene identified in the Pandoraea plasmids, may act in a similar way. The endonuclease genes found in pPF72-2, pPO70-1 and pPV15, share similarity to the plasmid conjugative transfer endonuclease encoding gene of Collimonas arenae $(60 \%$ identity), Burkholderia sp. AU4i (69\% identity) and Burkholderia cenocepacia H111 (69\% identity), respectively.

The conjugation region in pPF72-1, pPF72-2, pPO70-1, pPO70-2 and pPV15 is presented in Figure 1 as the only significant region that is shared amongst the Pandoraea plasmids. The shared region contains the same conjugation genes except for traD, which is only present in pPO70-1, pPO70-2 and pPV15. Gene arrangements in this region appear to be conserved amongst the five plasmids. Pairwise comparisons between the conjugation regions yielded $88-100 \%$ nucleotide identity values shared between the respective genes, further leading to the possibility of common origin for these regions.

\section{Other Genes}

\section{Virulence and Antibiotic Resistance Genes}

One of the important features of plasmids is the capability of carrying genes conferring virulence and antibiotic resistance properties, and transmitting these properties to new host strains. To explore the virulence and antibiotic resistance properties delivered by Pandoraea plasmids, we identified putative virulence and antibiotic resistance genes based on the search against

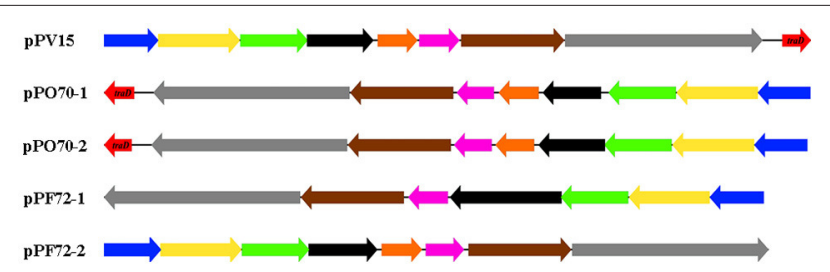

FIGURE 1 | Genetic arrangement of the shared conjugation region of plasmids pPF72-1, pPF2-2, pPO70-1, pPO70-2 and pPV15. Predicted conjugation genes and their orientation are presented in block arrows. The different genes are represented in colors: virB9 (blue), type VI secretion system (T6SS) protein-encoding gene (yellow), virB11 (green), lytic transglycosylase protein encoding gene (black), hypothetical protein encoding gene (orange), endonuclease protein encoding gene (pink), trwB (brown), relaxase protein encoding gene (gray), traD (red). Difference in orientation of the conjugation regions amongst the plasmids are represented by the direction of the block arrows. Block arrows pointing toward the left indicate reverse strand orientation whereas block arrows pointing toward the right indicate forward strand orientation.
VFDB (Chen et al., 2005) (Supplementary Table S4) and CARD (McArthur et al., 2013) (Supplementary Table S5), respectively, employing a cutoff value of $40 \%$ identity and $50 \%$ coverage.

All eight Pandoraea plasmids were found to carry putative virulence genes with a total ranging from 3 to 91 . The genes mainly encode functions related to type II (T2SS), III (T3SS), and IV (T4SS) secretion systems, purine biosynthesis, iron acquisition, DNA repair, replication and recombination, adherence, immune evasion, surface protein, motility, ureolytic and sialidase activities.

Plasmid pPA35 carries virulence genes encoding functions involved in T4SS ( $p i l D$, lepB). A previous study by Paranjpye et al. (1998), showed that mutation in pilD caused a defect in pilus expression and protein transport using the type II secretion pathway. This has led to a reduced adherence to epithelial tissue culture, cytotoxic activity and virulence. The lepB gene, encoding a SPAase homolog, is known to be necessary for viability (Waite et al., 2012). A purC gene encoding phosphoribosylaminoimidazole-succinocarboxamide synthetase involved in purine biosynthesis, has also been predicted in pPA35. Mutation in this gene was found to minimize cytotoxicity and formation of biofilm in the infection-causing bacterium, Pseudomonas aeruginosa, among CF patients (Guo et al., 2016). In addition, Jackson et al. (1999) presented that mutation of purC affected the growth ability of $M$. tuberculosis and M. bovis BCG in mouse bone marrow macrophages.

Compared to the number of virulence genes observed in pPA35, the plasmids of non-clinical host strains harbor more virulence genes, which will enable the respective host strains to adapt to their constantly changing environment. Besides that, in the event that these host strains are transmitted into a clinical setting, they may confer pathogenicity. Majority of the virulence genes in plasmids from non-clinical strains encode transposases, indicating the means of adaptive evolution of the plasmids. Other virulence genes that were mostly observed as well, are those which encode functions related to T3SS and T4SS.

Plasmid pPF72-1 carries virulence genes encoding functions related to T3SS (ssph2, ssaJ, escN), T4SS (pilB), motility $(f \lg M)$, iron acquisition $(e n t D, h \times u B)$ and cell surface $(b c a)$. Virulence gene ssph2 is a Salmonella typhimurium gene, that has participated in causing virulence in calves (Miao et al., 1999) whereas ssaJ, which encodes a T3SS component of Salmonella pathogenicity island (SPI-2), was found to possibly lead to reduction of virulence upon a transposon insertion in the gene (Hensel et al., 1997). The escN gene on the other hand encodes the T3SS ATPase of Escherichia coli (Andrade et al., 2007), which together with proton motive force, provides energy for secretion (Minamino and Namba, 2008). Assembly ATPase of the T4SS pilus encoded by pilB, functions in giving rise to virulence upon its encounter with host tissues (Persat et al., 2015). Gene flgM, which encodes a negative regulator of flagellin synthesis, causes depletion of $S$. typhimurium in a typhoid fever mouse model upon mutation (Schmitt et al., 1994). Iron acquisition gene entD resulted in virulence termination of extra-intestinal pathogenic E. coli (ExPEC) upon inactivation (Martin et al., 2013) whereas $h x u B$ is part of the haem-haemopexin utilization gene cluster with a role in virulence (Morton et al., 2007). Lastly, bca, 
which encodes for $\mathrm{C}$ protein alpha-antigen, causes a decrease in bacterial virulence upon knock-out mutation and is significant in group B Streptococcus pathogenicity (Li et al., 1997).

Genes encoding functions related to T4SS components (trwD, BMEII0028-encodes a VirB4 ATPase homolog) (VayssierTaussat et al., 2010; Wallden et al., 2010)and DNA repair, replication and recombination $(s s b)$, were identified in pPF72-2. The $s s b$ gene encodes SSB, which controls the activity of RecBCD nuclease as reported in E. coli (Anderson and Kowalczykowski, 1998). Loss of function of this nuclease will lead to a decrease in virulence as observed in S. enterica (Cano et al., 2002).

Only T3SS component encoding genes (ssaT, bscj, escS) (Hensel et al., 1997 and Tomich et al., 2003) have been identified in pPO70-1. A previous study by Deiwick et al. (1998) showed that mutation in ssaT caused a decrease in gene expression of S. typhimurium pathogenicity island, SP1. Additionally, sensitivity to gentamicin and polymyxin $\mathrm{B}$ antibiotics also resulted from the mutation. The protein encoded by $b s c j$ forms the T3SS channel, which functions to secrete effector proteins (Plano et al., 2001). Virulence gene escS on the other hand, encodes the T3SS component of enteropathogenic E. coli. Besides that, several putative genes encoding proteins involved in the production of urease and its activation were discovered in pPO70-1. These genes encode urease subunits alpha, beta and gamma, as well as urease accessory proteins UreD, UreE, UreF, and UreG, which are all involved ureolytic activity (Koper et al., 2004). Products of ureolytic activity are exploited to maintain surrounding $\mathrm{pH}$ (Burton and Prosser, 2001). The presence of these genes will enable the host strain to adapt in a urea-rich environment. Besides that, the production of urease also contributes as a virulence factor among human pathogens such as Helicobacter pylori, as well as in fungi namely Cryptococcus neoformans and Coccidioides posadasii (Cox et al., 2000; Rutherford, 2014).

The T4SS component encoding gene BMEIIO025 (encodes an attachment mediating protein VirB1 homolog) (Wallden et al., 2010) has been identified in pPO70-2 along with an adherence gene (efal) and two sialidase (nanj) genes. Gene efal was identified in enterohaemorrhagic $E$. coli which was shown to play a part in cell adherence (Nicholls et al., 2000). Chiarezza et al. (2009) have described nanj to have effect in increasing alpha-toxin-mediated cytotoxicity, however is not essential for virulence relating to gas gangrene.

Two T4SS related genes (bepF) have been identified in pPO70-3 encoding a Bartonella $(B$.) effector protein, which in a previous study was translocated via $B$. henselae T4SS into infected endothelial cells (Schmid et al., 2004).

As compared to pPO70-1, pPO70-2 and pPO70-3, only pPO70-4 was found to contain a virulence factor encoding gene related toT2SS $(g s p D)$. The T2SS, also known as the general secretory pathway (GSP), has been previously reported to be involved in bacterial virulence (Iwobi et al., 2003; Baldi et al., 2012). The $g s p D$ gene encodes a protein that forms the pore of T2SS in the outer membrane. Virulence gene $\operatorname{pcrV}$ was also identified in pPO70-4, encoding an important component in Pseudomonas T3SS which plays a part in cytotoxicity (Nanao et al., 2003). Besides that, genes encoding components of T4SS
(trwD, trwM, BMEII0025-encodes an attachment mediating protein VirB1 homolog, BMEII0034-encodes a channel protein VirB10 homolog) have also been identified in pPO70-4 (VayssierTaussat et al., 2010; Wallden et al., 2010) along with $t v i B$ which encodes an enzyme contributing to virulence antigen $\mathrm{Vi}$ formation in S. typhi (Zhang et al., 2006).

The adherence gene $f n b B$ identified in pPV15, encodes fibronectin-binding protein $\mathrm{B}$ which was shown to be present in majority of $S$. aureus isolates from different infection types, indicating a contribution to virulence (Arciola et al., 2005).

Antibiotic resistance genes were predicted in all Pandoraea plasmids except in pPA35 and pPO70-3. These genes were predicted at a small number ranging from 1 to 6 with pPF72-2 and pPO70-2 having the least. The predicted genes confer resistance to tetracyclin, beta-lactamase, aminoglycoside, fluoroquinolone, chloramphenicol, lincosamide, and macrolide resistance.

Genes encoding multidrug ABC transporter ATPases were observed in plasmids pPF72-1, pPO70-1 and pPV15. This subunit, which forms part of the $\mathrm{ABC}$ transporter, functions in promoting the transport of cytotoxic drugs across the membrane. Tetracycline resistance genes tet $M$ and tet36 have been identified in pPV15 and pPO70-1, respectively, where both encode ribosomal protection proteins (Whittle et al., 2003; Dönhöfer et al., 2012). Genes encoding proteins involved in betalactamase resistance, FEZ-1 beta-lactamase (blaFEZ-1) (Mercuri et al., 2001) and AQU-1-type AmpC beta-lactamase (PérezPérez and Hanson, 2002), were identified in pPO70-1 and pPO70-4, respectively. On the other hand, genes conferring resistance to aminoglycoside ( $r p h)$ (Shaw et al., 1993) and fluoroquinolone $(\mathrm{gyrB})$ (Nasri Yaiche et al., 2014), were found in pPF72-2 and pPO70-1, respectively. Additionally, genes involved in macrolide (mgt) (Quirós et al., 2000) and chloramphenicol (cmlA4) (Poirel et al., 2000) resistance were also identified in pPO70-1. Lincosamide resistance gene $(\ln u B)$ (Almuzara et al., 2013) was found in pPO70-2 whereas pPO70-4 contains two genes $(\mathrm{mel})$ that are associated with macrolide resistance (Ambrose et al., 2005).

It is observed that antibiotic resistance genes were detected only in plasmids from non-clinical Pandoraea strains which have not been isolated previously from any clinical source. We hypothesize that these plasmids convey the respective host strains with antibiotic resistance properties that are essential to survive in their respective non-clinical habitats. In the event that the strains are transmitted into clinical settings, the antibiotic resistance genes encoded by the plasmids would perhaps be able to equip them with persistence against antimicrobial treatment. However, more work is required to prove this speculation.

\section{CONCLUSION}

Eight complete plasmid sequences in Pandoraea were characterized and comparatively studied for the first time. These novel plasmids were successfully characterized according to the plasmid maintenance systems (replication, segregation, TA systems), conjugation, virulence and antibiotic resistance 
categories. Diverse and interesting features have been observed in the plasmids which include the possibility of host machinery interaction in plasmid segregation and replication. The characterization of the plasmid maintenance system according to replication, segregation and TA systems is important as we will not only gain insights into the mechanisms of plasmid survival in Pandoraea, but also perhaps aid in the treatment of Pandoraea pathogenicity. The mechanisms of plasmid maintenance can serve as targets in drug design, in order to prevent the continuous presence of virulent plasmids through cell generations. The knowledge of plasmid self-transmission through conjugation genes identification would be useful in the treatment of diseases especially when the self-transmitted plasmid itself carries virulence and antibiotic resistance genes. Other than being equipped with pathogenicity characteristics, plasmids with genes related to virulence and antibiotic resistance, provide the respective host strains with an advantage in adapting to their environment. Future studies should be carried out in confirming the functions of the predicted genes and discovering any hidden functional mechanisms.

\section{AUTHOR CONTRIBUTIONS}

DY, KKT, WFY, and KGC carried out data collection and experiments. KGC conceived the idea and obtained funding. WFY handled the project finance. DY performed the analyses, interpretation of data and wrote the manuscript. All authors proofread and approved the final version of the manuscript.

\section{ACKNOWLEDGMENTS}

This research was supported by the High Impact Research Grants, University of Malaya (UM-MOHE HIR Grant UM.C/ 625/1/HIR/MOHE/CHAN/14/1, Grant No. H-50001-A000027; UM-MOHE HIR Grant UM.C/625/1/HIR/MOHE/CHAN/01, Grant No. A-000001-50001) awarded to KGC which are gratefully acknowledged. DY thanks the financial support from University of Malaya for the Postgraduate Research Fund (PPP) (Grant number: PG138-2016A). The authors would like to thank Robson Ee and Lim Yan Lue for their laboratory assistance.

\section{SUPPLEMENTARY MATERIAL}

The Supplementary Material for this article can be found online at: http://journal.frontiersin.org/article/10.3389/fmicb. 2016.01606

Supplementary Figure S1 | Plasmids found in $P$. faecigallinarum DSM

23572 ${ }^{\top}$ : (A) pPF72-1 and (B) pPF72-1. Genes located on the positive strand are drawn on the outside of the plasmid circle whereas genes located on the negative strand are drawn on the inside: conjugation genes (pink); replication genes (dark blue); partitioning genes (green); TA genes (red); virulence genes (yellow); antibiotic resistance genes (light blue); others (gray).

Supplementary Figure S2 | Plasmids found in $P$. oxalativorans DSM $23570^{\top}$ : (A) pPO70-1, (B) pPO70-2, (C) pPO70-3 and (D) pPO70-4. Genes located on the positive strand are drawn on the outside of the plasmid circle whereas genes located on the negative strand are drawn on the inside: conjugation genes (pink); replication genes (dark blue); partitioning genes (green); TA genes (red); virulence genes (yellow); antibiotic resistance genes (light blue); others (gray).

Supplementary Figure S3 | Plasmid pPV15 from $P$. vervacti NS15. Genes located on the positive strand are drawn on the outside of the plasmid circle whereas genes located on the negative strand are drawn on the inside: conjugation genes (pink); virulence genes (yellow); antibiotic resistance genes (light blue); others (gray).

Supplementary Figure S4 | Plasmid pPA35 from P. apista DSM $16535^{\top}$. Genes located on the positive strand are drawn on the outside of the plasmid circle whereas genes located on the negative strand are drawn on the inside: conjugation genes (pink); replication genes (dark blue); partitioning genes (green); TA genes (red); virulence genes (yellow); others (gray).

Supplementary Figure S5 | GC-skew plots: (A) pPF72-1; (B) pPF72-2. The oriV is indicated by a minimum value of the cumulative GC-skew whereas terV is indicated by a maximum value of the cumulative GC-skew.

Supplementary Figure S6 | GC-skew plots: (A) pPO70-1; (B) pPO70-2; (C) pPO70-3; (D) pPO70-4. The oriV is indicated by a minimum value of the cumulative GC-skew whereas terV is indicated by a maximum value of the cumulative GC-skew.

Supplementary Figure S7 | GC-skew plot: pPV15. The oriV is indicated by a minimum value of the cumulative GC-skew whereas terV is indicated by a maximum value of the cumulative GC-skew.

Supplementary Figure S8 | GC-skew plot: pPA35. The oriV is indicated by a minimum value of the cumulative GC-skew whereas terV is indicated by a maximum value of the cumulative GC-skew.

Supplementary Table S1 | Lists of putative protein-encoding genes. Putative protein-encoding genes were highlighted according to replication (pink), segregation (green), conjugation (blue), ureolytic activity (yellow) and multidrug ABC transporter ATPase (orange) categories.

Supplementary Table S2 | List of putative toxin-antitoxin (TA) systems.

Supplementary Table S3 | Conjugation information of additional genes in pPA35 and pPO70-4. Yellow highlights indicate TA systems with toxin gene located upstream of the antitoxin gene.

Supplementary Table S4 | Lists of putative virulence genes. Genes were highlighted according to the different functions: Type II secretion system (emerald), Type III secretion system (blue), Type IV secretion system (green), purine biosynthesis (peach), motility (yellow), iron acquisition (brown), cell surface (pink), DNA repair, replication and recombination (orange), adherence (gray), sialidase activity (red).

Supplementary Table S5 | List of putative resistance genes. Genes were highlighted according to the different resistance functions: Multidrug ABC transporter ATPase (blue), tetracycline (green), beta-lactamase (pink), aminoglycoside (orange), fluoroquinolone (gray), macrolide (yellow), chloramphenicol (brown), lincosamide (emerald).

\section{REFERENCES}

Agnoli, K., Frauenknecht, C., Freitag, R., Schwager, S., Jenul, C., Vergunst, A., et al. (2014). The third replicon of members of the Burkholderia cepacia complex, plasmid pC3, plays a role in stress tolerance. Appl. Environ. Microbiol. 80, 1340-1348. doi: 10.1128/AEM.03 $330-13$

Almuzara, M., Bonofiglio, L., Cittadini, R., Vera Ocampo, C., Montilla, A., Del Castillo, M., et al. (2013). First case of Streptococcus lutetiensis bacteremia involving a clindamycin-resistant isolate carrying the lnuB gene. J. Clin. Microbiol. 51, 4259-4261. doi: 10.1128/JCM.01774-13

Alt-Mörbe, J., Stryker, J. L., Fuqua, C., Li, P. L., Farrand, S. K., and Winans, S. C. (1996). The conjugal transfer system of Agrobacterium tumefaciens octopine-type $\mathrm{Ti}$ plasmids is closely related to the transfer system of an 
IncP plasmid and distantly related to Ti plasmid vir genes. J. Bacteriol. 178, 4248-4257.

Ambrose, K. D., Nisbet, R., and Stephens, D. S. (2005). Macrolide efflux in Streptococcus pneumoniaeis mediated by a dual efflux pump ( $m e l$ and $m e f$ ) and is erythromycin inducible. Antimicrob. Agents Chemother. 49, 4203-4209. doi: 10.1128/AAC.49.10.4203-4209.2005

Anandham, R., Indiragandhi, P., Kwon, S. W., Sa, T. M., Jeon, C. O., Kim, Y. K., et al. (2010). Pandoraea thiooxydans sp. nov., a facultatively chemolithotrophic, thiosulfate-oxidizing bacterium isolated from rhizosphere soils of sesame (Sesamum indicum L.). Int. J. Syst. Evol. Microbiol. 60, 21-26. doi: 10.1099/ijs.0.012823-0

Anderson, D. G., and Kowalczykowski, S. C. (1998). SSB protein controls RecBCD enzyme nuclease activity during unwinding: a new role for looped intermediates. J. Mol. Biol. 282, 275-285. doi: 10.1006/jmbi.1998.2013

Andrade, A., Pardo, J. P., Espinosa, N., Pérez-Hernández, G., and GonzálezPedrajo, B. (2007). Enzymatic characterization of the enteropathogenic Escherichia coli type III secretion ATPase EscN. Arch. Biochem. Biophys. 468, 121-127. doi: 10.1016/j.abb.2007.09.020

Arciola, C. R., Campoccia, D., Gamberini, S., Baldassarri, L., and Montanaro, L. (2005). Prevalence of $c n a f n b A$ and $f n b B$ adhesin genes among Staphylococcus aureusisolates from orthopedic infections associated to different types of implant. FEMS Microbiol. Lett. 246, 81-86. doi: 10.1016/j.femsle.2005.03.035

Baldi, D. L., Higginson, E. E., Hocking, D. M., Praszkier, J., Cavaliere, R., James, C. E., et al. (2012). The type II secretion system and its ubiquitous lipoprotein substrate, SsIE, are required for biofilm formation and virulence of enteropathogenic Escherichia coli. Infect. Immun. 80, 2042-2052. doi: 10.1128/IAI.06160-11

Baxter, J. C. and Funnell, B. E. (2014). Plasmid partition mechanisms. Microbiol. Spectr. 2:PLAS-0023-2014. doi: 10.1128/microbiolspec.PLAS-0023-2014

Beceiro, A., Tomás, M., and Bou, G. (2013). Antimicrobial resistance and virulence: a successful or deleterious association in the bacterial world? Clin. Microbiol. Rev. 26, 185-230. doi: 10.1128/CMR.00059-12

Bignell, C., and Thomas, C. M. (2001). The bacterial ParA-ParB partitioning proteins. J. Biotechnol. 91, 1-34. doi: 10.1016/S0168-1656(01)00293-0

Budde, P. P., Davis, B. M., Yuan, J., and Waldor, M. K. (2007). Characterization of a higBA toxin-antitoxin locus in Vibrio cholerae. J. Bacteriol. 189, 491-500. doi: 10.1128/JB.00909-06

Burrus, V., and Waldor, M. K. (2004). Shaping bacterial genomes with integrative and conjugative elements. Res. Microbiol. 155, 376-386. doi: 10.1016/j.resmic. 2004.01.012

Burton, S. A. Q., and Prosser, J. I. (2001). Autotrophic ammonia oxidation at low ph through urea hydrolysis. Appl. Environ. Microbiol. 67, 2952-2957. doi: 10.1128/AEM.67.7.2952-2957.2001

Bustamante, P., Tello, M., and Orellana, O. (2014). Toxin-antitoxin systems in the mobile genome of Acidithiobacillus ferrooxidans. PLoS ONE 9:e112226. doi: 10.1371/journal.pone.0112226

Cano, D. A., Pucciarelli, M. G., García-del Portillo, F., and Casadesús, J. (2002). Role of the RecBCD recombination pathway in Salmonella virulence. $J$. Bacteriol. 184, 592-595. doi: 10.1128/JB.184.2.592-595.2002

Chan, K.-G., Yong, D., Ee, R., Lim, Y.-L., Yu, C.-Y., Tee, K.-K., et al. (2016). Complete genome sequence of Pandoraea oxalativorans DSM $23570^{\mathrm{T}}$, an oxalate metabolizing soil bacterium. J. Biotechnol. 219, 124-125. doi: 10.1016/ j.jbiotec.2015.12.037

Chan, W. T., Moreno-Córdoba, I., Yeo, C. C., and Espinosa, M. (2012). Toxinantitoxin genes of the Gram-positive pathogen Streptococcus pneumoniae: so few and yet so many. Microbiol. Mol. Biol. Rev. 76, 773-791. doi: 10.1128/ MMBR.00030-12

Chen, L., Yang, J., Yu, J., Yao, Z., Sun, L., Shen, Y., et al. (2005). VFDB: a reference database for bacterial virulence factors. Nucleic Acids Res. 33, D325-D328. doi: 10.1093/nar/gki008

Chiarezza, M., Lyras, D., Pidot, S. J., Flores-Díaz, M., Awad, M. M., Kennedy, C. L., et al. (2009). The NanI and NanJ sialidases of Clostridium perfringensare not essential for virulence. Infect. Immun. 77, 4421-4428. doi: 10.1128/IAI. 00548-09

Christensen-Dalsgaard, M., Jørgensen, M. G., and Gerdes, K. (2010). Three new RelE-homologous mRNA interferases of Escherichia coli differentially induced by environmental stresses. Mol. Microbiol. 75, 333-348. doi: 10.1111/j.13652958.2009.06969.x
Christie, P. J. (1997). Agrobacterium tumefaciens T-complex transport apparatus: a paradigm for a new family of multifunctional transporters in eubacteria. $J$. Bacteriol. 179, 3085-3094.

Coenye, T., Falsen, E., Hoste, B., Ohlén, M., Goris, J., Govan, J. R., et al. (2000). Description of Pandoraea gen. nov. with Pandoraea apista sp. nov., Pandoraea pulmonicola sp. nov., Pandoraea pnomenusa sp. nov., Pandoraea sputorum sp. nov. and Pandoraea norimbergensis comb. nov. Int. J. Syst. Evol. Microbiol. 50, 887-899. doi: 10.1099/00207713-50-2-887

Cox, G. M., Mukherjee, J., Cole, G. T., Casadevall, A., and Perfect, J. R. (2000). Urease as a virulence factor in experimental cryptococcosis. Infect. Immun. 68, 443-448. doi: 10.1128/IAI.68.2.443-448.2000

Deiwick, J., Nikolaus, T., Shea, J. E., Gleeson, C., Holden, D. W., and Hensel, M. (1998). Mutations in Salmonellapathogenicity island 2 (SPI2) genes affecting transcription of SPI1 genes and resistance to antimicrobial agents. J. Bacteriol. $180,4775-4780$.

del Solar, G., Giraldo, R., Ruiz-Echevarría, M. J., Espinosa, M., and Díaz-Orejas, R. (1998). Replication and control of circular bacterial plasmids. Microbiol. Mol. Biol. Rev. 62, 434-464.

Dönhöfer, A., Franckenberg, S., Wickles, S., Berninghausen, O., Beckmann, R., and Wilson, D. N. (2012). Structural basis for TetM-mediated tetracycline resistance. Proc. Natl. Acad. Sci. U.S.A. 109, 16900-16905. doi: 10.1073/pnas.1208037109

Ee, R., Yong, D., Lim, Y. L., Yin, W.-F., and Chan, K.-G. (2015). Complete genome sequence of oxalate-degrading bacterium Pandoraea vervacti DSM $23571^{\mathrm{T}}$. J. Biotechnol. 204, 5-6. doi: 10.1016/j.jbiotec.2015.03.020

Fernández-Tresguerres, M. E., Martín, M., García de Viedma, D., Giraldo, R., and Díaz-Orejas, R. (1995). Host growth temperature and a conservative amino acid substitution in the replication protein of pPS10 influence plasmid host range. J. Bacteriol. 177, 4377-4384.

Gerdes, K., Møller-Jensen, J., and Bugge Jensen, R. (2000). Plasmid and chromosome partitioning: surprises from phylogeny. Mol. Microbiol. 37, 455-466. doi: 10.1046/j.1365-2958.2000.01975.x

Gillespie, J. J., Beier, M. S., Rahman, M. S., Ammerman, N. C., Shallom, J. M., Purkayastha, A., et al. (2007). Plasmids and Rickettsial evolution: insight from Rickettsia felis. PLoS ONE 2:e266. doi: 10.1371/journal.pone.0000266

Grigoriev, A. (1998). Analyzing genomes with cumulative skew diagrams. Nucleic Acids Res. 26, 2286-2290. doi: 10.1093/nar/26.10.2286

Guo, Q., Wei, Y., Xia, B., Jin, Y., Liu, C., Pan, X., et al. (2016). Identification of a small molecule that simultaneously suppresses virulence and antibiotic resistance of Pseudomonas aeruginosa. Sci. Rep. 6:19141. doi: 10.1038/srep 19141

Guynet, C., Cuevas, A., Moncalián, G., and de la Cruz, F. (2011). The stb operon balances the requirements for vegetative stability and conjugative transfer of plasmid R388. PLoS Genet. 7:e1002073. doi: 10.1371/journal.pgen.1002073

Hellyer, T. J., Brown, I. N., Dale, J. W., and Easmon, C. S. (1991). Plasmid analysis of Mycobacterium avium-intracellulare (MAI) isolated in the United Kingdom from patients with and without AIDS. J. Med. Microbiol. 34, 225-231. doi: 10.1099/00222615-34-4-225

Hensel, M., Shea, J. E., Raupach, B., Monack, D., Falkow, S., Gleeson, C., et al. (1997). Functional analysis of ssaJ and the ssaK/U operon, 13 genes encoding components of the type III secretion apparatus of Salmonella pathogenicity island 2. Mol. Microbiol. 24, 155-167. doi: 10.1046/j.1365-2958.1997.3271699.x

Hyatt, D., Chen, G. L., LoCascio, P. F., Land, M. L., Larimer, F. W., and Hauser, L. J. (2010). Prodigal: prokaryotic gene recognition and translation initiation site identification. BMC Bioinform. 11:119. doi: 10.1186/1471-2105-11-119

Iwobi, A., Heesemann, J., Garcia, E., Igwe, E., Noelting, C., and Rakin, A. (2003). Novel virulence-associated type II secretion system unique to highpathogenicity Yersinia enterocolitica. Infect. Immun. 71, 1872-1879. doi: 10.1128/IAI.71.4.1872-1879.2003

Jackson, M., Phalen, S. W., Lagranderie, M., Ensergueix, D., Chavarot, P., Marchal, G., et al. (1999). Persistence and protective efficacy of a Mycobacterium tuberculosis auxotroph vaccine. Infect. Immun. 67, 2867-2873.

Koper, T. E., El-Sheikh, A. F., Norton, J. M., and Klotz, M. G. (2004). Ureaseencoding genes in ammonia-oxidizing bacteria. Appl. Environ. Microbiol. 70, 2342-2348. doi: 10.1128/AEM.70.4.2342-2348.2004

Kroll, J., Klinter, S., Schneider, C., Voß, I., and Steinbüchel, A. (2010). Plasmid addiction systems: perspectives and applications in biotechnology. Microb. Biotechnol. 3, 634-657. doi: 10.1111/j.1751-7915.2010.00170.x 
Krumsiek, J., Arnold, R., and Rattei, T. (2007). Gepard: a rapid and sensitive tool for creating dotplots on genome scale. Bioinformatics 23, 1026-1028. doi: 10.1093/bioinformatics/btm039

Li, J., Kasper, D. L., Ausubel, F. M., Rosner, B., and Michel, J. L. (1997). Inactivation of the $\alpha \mathrm{C}$ protein antigen gene, $b c a$, by a novel shuttle/suicide vector results in attenuation of virulence and immunity in group B Streptococcus. Proc. Natl. Acad. Sci. U.S.A. 94, 13251-13256.

Lim, Y.-L., Ee, R., Yin, W.-F., and Chan, K.-G. (2014). Quorum sensing activity of Aeromonas Caviae strain YL12, a bacterium isolated from compost. Sensors 14, 7026-7040. doi: 10.3390/s140407026

Lim, Y.-L., Ee, R., Yong, D., Yu, C.-Y., Ang, G.-Y., Tee, K.-K., et al. (2016). Complete genome sequence analysis of Pandoraea pnomenusa type strain DSM $16536^{\mathrm{T}}$ isolated from a cystic fibrosis patient. Front. Microbiol. 7:109. doi: 10.3389/fmicb.2016.00109

Lujan, S. A., Guogas, L. M., Ragonese, H., Matson, S. W., and Redinbo, M. R. (2007). Disrupting antibiotic resistance propagation by inhibiting the conjugative DNA relaxase. Proc. Natl. Acad. Sci. U.S.A. 104, 12282-12287. doi: $10.1073 /$ pnas.0702760104

Mahenthiralingam, E., Baldwin, A., and Dowson, C. G. (2008). Burkholderia cepacia complex bacteria: opportunistic pathogens with important natural biology. J. Appl. Microbiol. 104, 1539-1551. doi: 10.1111/j.13652672.2007.03706.x

Marchler-Bauer, A., Derbyshire, M. K., Gonzales, N. R., Lu, S., Chitsaz, F., Geer, L. Y., et al. (2015). CDD: NCBI's conserved domain database. Nucleic Acids Res. 43, D222-D226. doi: 10.1093\%2Fnar\%2Fgku1221

Martin, P., Marcq, I., Magistro, G., Penary, M., Garcie, C., Payros, D., et al. (2013). Interplay between siderophores and colibactin genotoxin biosynthetic pathways in Escherichia coli. PLoS Pathog. 9:e1003437. doi: 10.1371/journal. ppat. 1003437

McArthur, A. G., Waglechner, N., Nizam, F., Yan, A., Azad, M. A., Baylay, A. J., et al. (2013). The comprehensive antibiotic resistance database. Antimicrob. Agents Chemother. 57, 3348-3357. doi: 10.1128/AAC.00419-13

Mercuri, P. S., Bouillenne, F., Boschi, L., Lamotte-Brasseur, J., Amicosante, G., Devreese, B., et al. (2001). Biochemical characterization of the FEZ-1 metallo$\beta$-lactamase of Legionella gormanii ATCC $33297^{\mathrm{T}}$ produced in Escherichia coli. Antimicrob. Agents Chemother. 45, 1254-1262. doi: 10.1128/AAC.45.4.12541262.2001

Meyer, R. R., and Laine, P. S. (1990). The single-stranded DNA-binding protein of Escherichia coli. Microbiol. Rev. 54, 342-380.

Miao, E. A., Scherer, C. A., Tsolis, R. M., Kingsley, R. A., Adams, L. G., Bäumler, A. J., et al. (1999). Salmonella typhimurium leucine-rich repeat proteins are targeted to the SPI1 and SPI2 type III secretion systems. Mol. Microbiol. 34, 850-864. doi: 10.1046/j.1365-2958.1999.01651.x

Minamino, T., and Namba, K. (2008). Distinct roles of the FliI ATPase and proton motive force in bacterial flagellar protein export. Nature 451, 485-488. doi: 10.1038 /nature 06449

Morton, D. J., Seale, T. W., Madore, L. L., VanWagoner, T. M., Whitby, P. W., and Stull, T. L. (2007). The haem-haemopexin utilization gene cluster $(h x u C B A)$ as a virulence factor of Haemophilus influenzae. Microbiology 153, 215-224. doi: 10.1099/mic.0.2006/000190-0

Nanao, M., Ricard-Blum, S., Di Guilmi, A. M., Lemaire, D., Lascoux, D., Chabert, J., et al. (2003). Type III secretion proteins PcrV and PcrG from Pseudomonas aeruginosa form a 1:1 complex through high affinity interactions. BMC Microbiol. 3:21. doi: 10.1186/1471-2180-3-21

Nasri Yaiche, M., Denden Rafraf, I., Guo, Q., Mastouri, M., Aouni, M., and Wang, M. (2014). Type II and type IV topoisomerase mutations in clinical isolates of Morganella morganii harbouring the qnrD gene. Ann. Clin. Microbiol. Antimicrob.13, 34. doi: 10.1186/s12941-014-0034-4

Nicholls, L., Grant, T. H., and Robins-Browne, R. M. (2000). Identification of a novel genetic locus that is required for in vitro adhesion of a clinical isolate of enterohaemorrhagic Escherichia coli to epithelial cells. Mol. Microbiol. 35, 275-288. doi: 10.1046/j.1365-2958.2000.01690.x

Pandey, D. P., and Gerdes, K. (2005). Toxin-antitoxin loci are highly abundant in free-living but lost from host-associated prokaryotes. Nucleic Acids Res. 33, 966-976. doi: 10.1093/nar/gki201

Paranjpye, R. N., Lara, J. C., Pepe, J. C., Pepe, C. M., and Strom, M. S. (1998). The type IV leader peptidase/N-methyltransferase of Vibrio vulnificus controls factors required for adherence to HEp- 2 cells and virulence in iron-overloaded mice. Infect. Immun. 66, 5659-5668.

Pérez-Pérez, F. J., and Hanson, N. D. (2002). Detection of plasmid-mediated AmpC $\beta$-lactamase genes in clinical isolates by using multiplex PCR. J. Clin. Microbiol. 40, 2153-2162. doi: 10.1128/JCM.40.6.2153-2162.2002

Persat, A., Inclan, Y. F., Engel, J. N., Stone, H. A., and Gitai, Z. (2015). Type IV pili mechanochemically regulate virulence factors in Pseudomonas aeruginosa. Proc. Natl. Acad. Sci. U.S.A. 112, 7563-7568. doi: 10.1073/pnas.1502025112

Picardeau, M., Lobry, J. R., and Hinnebusch, B. J. (2000). Analyzing DNA strand compositional asymmetry to identify candidate replication origins of Borrelia burgdorferilinear and circular plasmids. Genome Res. 10, 1594-1604. doi: 10.1101/gr.124000

Pimentel, J. D., and MacLeod, C. (2008). Misidentification of Pandoraea sputorum isolated from sputum of a patient with cystic fibrosis and review of Pandoraea species infections in transplant patients. J. Clin. Microbiol. 46, 3165-3168. doi: 10.1128/JCM.00855-08

Plano, G. V., Day, J. B., and Ferracci, F. (2001). Type III export: new uses for an old pathway. Mol. Micrbiol. 40, 284-293. doi: 10.1046/j.1365-2958.2001.02354.x

Poirel, L., Le Thomas, I., Naas, T., Karim, A., and Nordmann, P. (2000). Biochemical sequence analyses of GES-1, a novel class A extended-spectrum $\beta$-lactamase, and the class 1 integron In52 from Klebsiella pneumoniae. Antimicrob. Agents Chemother.44, 622-632. doi: 10.1128/aac.44.3.622-632.2000

Quevillon, E., Silventoinen, V., Pillai, S., Harte, N., Mulder, N., Apweiler, R., et al. (2005). InterProScan: protein domains identifier. Nucleic Acids Res. 33, W116-W120. doi: 10.1093/nar/gki442

Quirós, L. M., Carbajo, R. J., Brana, A. F., and Salas, J. A. (2000). Glycosylation of macrolide antibiotics. Purification and kinetic studies of a macrolide glycosyltransferase from Streptomyces antibioticus. J. Biol. Chem. 275, $11713-$ 11720. doi: 10.1074/jbc.275.16.11713

Rösch, T. C., Golman, W., Hucklesby, L., Gonzalez-Pastor, J. E., and Graumann, P. L. (2014). The Presence of conjugative plasmid pLS20 affects global transcription of its Bacillus subtilis host and confers beneficial stress resistance to cells. Appl. Environ. Microbiol. 80, 1349-1358. doi: 10.1128/AEM.03154-13

Rutherford, J. C. (2014). The emerging role of urease as a general microbial virulence factor. PLoS Pathog. 10:e1004062. doi: 10.1371/journal.ppat.1004062

Sahin, N., Tani, A., Kotan, R., Sedlácek, I., Kimbara, K., and Tamer, A. U. (2011) Pandoraea oxalativorans sp. nov., Pandoraea faecigallinarum sp. nov. and Pandoraea vervacti sp. nov., isolated from oxalate-enriched culture. Int. J. Syst. Evol. Microbiol. 61, 2247-2253. doi: 10.1099/ijs.0.026138-0

Schmid, M. C., Schulein, R., Dehio, M., Denecker, G., Carena, I., and Dehio, C. (2004). The VirB type IV secretion system of Bartonella henselae mediates invasion, proinflammatory activation and antiapoptotic protection of endothelial cells. Mol. Microbiol. 52, 81-92. doi: 10.1111/j.13652958.2003.03964.x

Schmitt, C. K., Darnell, S. C., Tesh, V. L., Stocker, B. A., and O’Brien, A. D. (1994). Mutation of flgM attenuates virulence of Salmonella typhimurium, and mutation of fliA represses the attenuated phenotype. J. Bacteriol. 176, 368-377.

Sengupta, M., and Austin, S. (2011). Prevalence and significance of plasmid maintenance functions in the virulence plasmids of pathogenic bacteria. Infect. Immun.79, 2502-2509. doi: 10.1128/IAI.00127-11

Shaw, K. J., Rather, P. N., Hare, R. S., and Miller, G. H. (1993). Molecular genetics of aminoglycoside resistance genes and familial relationships of the aminoglycoside-modifying enzymes. Microbiol. Rev. 57, 138-163.

Simpson, A. E., Skurray, R. A., and Firth, N. (2003). A single gene on the Staphylococcal multiresistance plasmid pSK1 encodes a novel partitioning system. J. Bacteriol. 185, 2143-2152. doi: 10.1128/JB.185.7.2143-2152.2003

Stolz, A. (2014). Degradative plasmids from sphingomonads. FEMS Microbiol. Lett. 350, 9-19. doi: 10.1111/1574-6968.12283

Stryjewski, M. E., LiPuma, J. J., Messier, R. H. Jr., Reller, L. B., and Alexander, B. D. (2003). Sepsis, multiple organ failure, and death due to Pandoraea pnomenusa infection after lung transplantation.J. Clin. Microbiol.41, 2255-2257. doi: 10.1128/JCM.41.5.2255-2257.2003

Sullivan, M. J., Zakour, N. B., Forde, B., Stanton-Cook, M., and Beatson, S. A. (2015). Contiguity: contig adjacency graph construction and visualisation. Peer J. PrePrints 3, e1273. doi: 10.7287/peerj.preprints.1037v1

Thomas, C. M. (2000). Paradigms of plasmid organization. Mol. Microbiol. 37, 485-491. doi: 10.1046/j.1365-2958.2000.02006.x 
Tomich, M., Griffith, A., Herfst, C. A., Burns, J. L., and Mohr, C. D. (2003). Attenuated virulence of a Burkholderia cepaciatype III secretion mutant in a murine model of infection. Infect. Immun. 71, 1405-1415. doi: 10.1128/IAI.71.3.1405-1415.2003

Unterholzner, S. J., Poppenberger, B., and Rozhon, W. (2013). Toxin-antitoxin systems. Mob. Genet. Elem. 3:e26219. doi: 10.4161/mge.26219

Van Melderen, L., and Saavedra De Bast, M. (2009). Bacterial toxinantitoxin systems: more than selfish entities? PLoS Genet. 5:e1000437. doi: 10.1371/journal.pgen.1000437

Vayssier-Taussat, M., Le Rhun, D., Deng, H. K., Biville, F., Cescau, S., Danchin, A., et al. (2010). The Trw type IV secretion system of Bartonella mediates host-specific adhesion to erythrocytes. PLoS Pathog. 6:e1000946. doi: 10.1371/journal.ppat.1000946

Venkatesan, M. M., Goldberg, M. B., Rose, D. J., Grotbeck, E. J., Burland, V., and Blattner, F. R. (2001). Complete DNA sequence and analysis of the large virulence plasmid of Shigella flexneri. Infect. Immun. 69, 3271-3285. doi: 10.1128/IAI.69.5.3271-3285.2001

Waite, R. D., Rose, R. S., Rangarajan, M., Aduse-Opoku, J., Hashim, A., and Curtis, M. A. (2012). Pseudomonas aeruginosa possesses two putative type I signal peptidases, LepB and PA1303, each with distinct roles in physiology and virulence. J. Bacteriol. 194, 4521-4536. doi: 10.1128/JB.06678-11

Wallden, K., Rivera-Calzada, A., and Waksman, G. (2010). Type IV secretion systems: versatility and diversity in function. Cell Microbiol. 12, 1203-1212. doi: 10.1111/j.1462-5822.2010.01499.x

Wang, A., and Macrina, F. L. (1995). Streptococcal plasmid pIP501 has a functional oriT site. J. Bacterilol. 177, 4199-4206

Whiley, H., Giglio, S., and Bentham, R. (2015). Opportunistic pathogens Mycobacterium Avium complex (MAC) and Legionella spp. colonise model shower. Pathogens 4, 590-598. doi: 10.3390/pathogens4030590

Whittle, G., Whitehead, T. R., Hamburger, N., Shoemaker, N. B., Cotta, M. A., and Salyers, A. A. (2003). Identification of a new ribosomal protection type of tetracycline resistance gene, tet(36), from swine manure pits Appl. Environ. Microbiol. 69, 4151-4158. doi: 10.1128/AEM.69.7.4151-41 58.2003

Yamaguchi, Y., Park, J. H., and Inouye, M. (2009). MqsR, a crucial regulator for quorum sensing and biofilm formation, is a GCU-specific mRNA interferase in Escherichia coli. J. Biol. Chem. 284, 28746-28753. doi: 10.1074/jbc.M109. 032904

Zhang, H., Zhou, Y., Bao, H., and Liu, H. (2006). Vi antigen biosynthesis in Salmonella typhi: characterization of UDP-N-acetylglucosamine C-6 dehydrogenase (TviB) and UDP-N-acetylglucosaminuronic acid C-4 epimerase (TviC). Biochemistry 45, 8163-8173. doi: 10.1021/bi060446d

Zheng, R., Zhang, Q., Guo, Y., Feng, Y., Liu, L., Zhang, A., et al. (2016). Outbreak of plasmid-mediated NDM-1-producing Klebsiella pneumoniae ST105 among neonatal patients in Yunnan, China. Ann. Clin. Microbiol. Antimicrob. 15, 10. doi: 10.1186/s12941-016-0124-6

Zienkiewicz, M., Kern-Zdanowicz, I., Gołębiewski, M., Żyliñska, J., Mieczkowski, P., Gniadkowski, M., et al. (2007). Mosaic structure of p1658/97, a 125kilobase plasmid harboring an active amplicon with the extended-spectrum $\beta$-Lactamase gene blaSHV-5. Antimicrob. Agents Chemother. 51, 1164-1171. doi: 10.1128/AAC.00772-06

Conflict of Interest Statement: The authors declare that the research was conducted in the absence of any commercial or financial relationships that could be construed as a potential conflict of interest.

Copyright (c) 2016 Yong, Tee, Yin and Chan. This is an open-access article distributed under the terms of the Creative Commons Attribution License (CC BY). The use distribution or reproduction in other forums is permitted, provided the original author(s) or licensor are credited and that the original publication in this journal is cited, in accordance with accepted academic practice. No use, distribution or reproduction is permitted which does not comply with these terms. 